\title{
Analysis and comparison of autologous platelet-rich plasma preparation systems used in the treatment of enthesopathies: A preliminary study
}

\author{
Maciej Dejnek ${ }^{1,2, A-F}$, Helena Moreira, $3, A-C$, Sylwia Płaczkowska ${ }^{4, B}$, Piotr Morasiewicz, ${ }^{5, A, E}$, \\ Ewa Barg ${ }^{3, A, E}$, Jarosław Witkowski ${ }^{1,2, D, E}$, Paweł Reichert ${ }^{1, A, E, F}$ \\ ${ }^{1}$ Department of Trauma and Hand Surgery, Wroclaw Medical University, Poland \\ 2 Department of Sports Medicine, Wroclaw Medical University, Poland \\ ${ }^{3}$ Department of Basic Medical Sciences, Wroclaw Medical University, Poland \\ ${ }^{4}$ Diagnostic Laboratory for Teaching and Research, Department of Laboratory Diagnostics, Wroclaw Medical University, Poland \\ ${ }^{5}$ Department of Orthopaedic and Trauma Surgery, Institute of Medical Sciences, University of Opole, Poland
}

A - research concept and design; B - collection and/or assembly of data; $C$ - data analysis and interpretation;

$D$ - writing the article; $E$ - critical revision of the article; $F$ - final approval of the article

Address for correspondence

Maciej Dejnek

E-mail:maciejdejnek@gmail.com

\section{Funding sources}

This research was financially supported by the Ministry of Health subvention according to number of STM.E067.20.112 from the IT Simple system of Wroclaw Medical University.

Conflict of interest

None declared

Received on December 30, 2020

Reviewed on February 6, 2021

Accepted on March 26, 2021

Published online on June 11, 2021

Cite as

Dejnek M, Moreira H, Płaczkowska S, et al. Analysis and comparison of autologous platelet-rich plasma preparation systems used in the treatment of enthesopathies: A preliminary study. Adv Clin Exp Med. 2021;30(7):757-764. doi:10.17219/acem/135045

DOI

10.17219/acem/135045

Copyright

Copyright by Author(s)

This is an article distributed under the terms of the

Creative Commons Attribution 3.0 Unported (CC BY 3.0)

(https://creativecommons.org/licenses/by/3.0/)

\begin{abstract}
Background. Autologous platelet-rich plasma (PRP) injection is an alternative but widely accepted method for the treatment of degenerative changes in tendon attachments known as enthesopathies. The PRP is considered a safe source for high concentrations of the growth factors involved in the healing process. Despite initial promising outcomes, many recent studies report conflicting results for this treatment. This may be due to differences in the concentrations of platelets and growth factors in PRPs obtained using different methods.

Objectives. The aim of this study was to compare PRP preparation systems in terms of morphotic components and selected growth factors to find the most appropriate procedure for the treatment of enthesopathies.

Materials and methods. Whole blood samples from 6 healthy male volunteers were collected. Using different commercial kits (Mini GPS III System, Arthrex ACP, and Xerthra, Dr. PRP), 4 PRPs were prepared from the blood of each participant. All samples were analyzed for the content of morphotic components and the following growth factors: transforming growth factor- $\beta 1$ (TGF- $\beta 1$ ), epidermal growth factor (EGF), vascular endothelial growth factor (VEGF), and platelet-derived growth factor AA (PDGF-AA).
\end{abstract}

Results. The Mini GPS III produced PRP with the highest concentration of platelets and white blood cells (WBC) compared to the other systems included in the study. Significant differences in the levels of EGF and PDGF-AA were found only between the Mini GPS III and Arthrex ACP. There was positive correlation between the content of platelets and the levels of PDGF-AA and EGF. The red blood cells (RBC) concentration positively correlated with PDGF-AA, EGF and VEGF.

Conclusions. This study showed differences between the morphotic components and levels of selected growth factors in PRP obtained with the different preparation methods. Due to insufficient data, we cannot argue for or against any of the studied protocols for the treatment of enthesopathy. Further studies on a larger population are required to validate our results.

Key words: platelet-rich plasma, growth factors, platelet-derived growth factor, enthesopathy 


\section{Background}

Enthesopathies are degenerative changes in the site of tendon attachment to the bone. This disease can affect many different sites in the human body. The most common and best described are changes in the attachment of wrist extensors or flexors to humeral epicondyles, the Achilles tendon, patellar tendon, suprascapular tendon, or plantar fascia. ${ }^{1}$ The main symptoms reported by patients include local pain and limitations in sport, work and daily activities. The cause of the disease still remains unclear. However, the most widely accepted theory is the accumulation of microinjuries resulting from repeated overloads that exceed the body's compensatory capacity. The change in the dogma of the inflammatory nature of this disease was the result of numerous histopathological studies that found disorganized tissue and neovessels within the involved tendon, but only few inflammatory cells. In many cases, symptoms resolve spontaneously and properly selected exercises help prevent them in the future. Unfortunately, some cases turn into a chronic condition that is very difficult to treat, and sometimes only surgical excision of the affected tissue can lead to improvement.

Due to the lack of effective therapy, many different methods have been proposed, and autologous platelet-rich plasma (PRP) is one of the most promising treatments. ${ }^{1-3}$ For this procedure, the patient's blood is collected and centrifuged to isolate the platelet-rich part of the plasma. This plasma is then administered into the affected tissue, typically by local injection. The $\alpha$-granules of platelets contain significant amounts of cytokines involved in tissue healing. ${ }^{4}$ It is expected that PRP containing a platelet concentration above the baseline will contain a significantly higher concentration of important cytokines, chemokines and growth factors. ${ }^{5}$ Numerous in vitro studies have shown that these biologically active components play a key role in tissue repair by stimulating proliferation, chemotaxis, cell differentiation, and angiogenesis. ${ }^{4,6,7}$

The acceleration of natural tissue healing processes by PRP administration was expected to revolutionize the treatment of injuries and chronic degenerative diseases like enthesopathy. Other diseases in which the natural balance between anabolic and catabolic processes is disrupted are also candidates for PRP treatment. Positive clinical outcomes have been reported for various conditions including bone nonunions, osteonecrosis, difficult-to-heal wounds, osteoarthritis, and sports injuries. ${ }^{8-12}$

The enthusiastic adoption of this method began to wane with the increasing appearance of studies showing conflicting results. ${ }^{12-14}$ A major problem with this body of research is the lack of a standardized definition for PRP. Many manufacturers have released commercially available kits for the easy preparation of PRP in an outpatient setting. ${ }^{8,15}$ These kits differ from each other in various parameters, such as the amount of material collected from the patient, the type of anticoagulant used, the structure of the separator, the length and speed of centrifugation, the method of extraction and activation, the assumed concentration of platelets and leukocytes in final product, and its consistency. ${ }^{16-19}$ These differences have made it almost impossible to compare the results of studies where PRP was prepared according to different protocols.

The answer to the above problem should be a reliable classification system for PRP. One of the classification systems designed to standardize the nomenclature is based on the content of fibrin and leukocytes in the PRP. In this system, 4 main classes of autologous PRPs are identified - PRP with a low content of leukocytes (P-PRP), PRP with a high content of leukocytes (L-PRP), platelet-rich fibrin with a low content of leukocytes (P-PRF), and plateletrich fibrin with a high content of leukocytes (L-PRF). ${ }^{20}$ However, these divisions do not include the concentration or the absolute number of platelets. Another classification system proposed to improve the comparison of results from different publications is the PAW system. This system is based on the 3 most important components of PRP: the absolute number of platelets, the method of their activation and the leukocyte content. ${ }^{21}$

The classification systems mentioned above do not solve all the problems connected with studies on PRP. The multiplicity of variables still makes it almost impossible to predict the content of different growth factors in PRP.

\section{Objectives}

The main goal of this study is to compare the PRP preparation systems available on the local market in terms of morphotic components and selected growth factors.

The results of such an analysis are essential for selecting the most appropriate procedure for daily clinical practice and for further research on the treatment of degenerative conditions. Regarding legal issues, only those systems that are officially registered for the treatment of enthesopathy were included in the study. However, our results can be helpful in decision-making for all conditions treated with PRP.

\section{Materials and methods}

\section{Study design and setting}

This controlled laboratory study was conducted at Wroclaw Medical University, Poland, in the Diagnostic Laboratory for Teaching and Research by clinicians and laboratory researchers. All procedures on human participants were conducted in accordance with the ethical standards of Wroclaw Medical University (Poland) and with the 1964 Helsinki Declaration and its later amendments. The study was approved by the local bioethics committee (Ethics Committee of Wroclaw Medical University, 30.03.2020, approval No. KB 163/2020). 


\section{Participants}

Six healthy male volunteers similar in age were asked for a whole blood donation. The number of participants included reflects the preliminary nature of the study. Criteria for inclusion in the study were an age of 27-28 years, absence of significant disease and conditions that could affect the blood morphotic components, at least 2 weeks without taking any drug that may interfere with the function of platelets, and a non-smoking status.

\section{Data sources and measurement}

Approximately $75 \mathrm{~mL}$ of whole blood was collected from each participant under aseptic conditions and immediately divided into 5 samples. First, $\sim 2 \mathrm{~mL}$ was transferred to a tube with ethylenediaminetetraacetic acid (EDTA) and then analyzed for morphotic components using an automatic laboratory analyzer (Mindray BC-5150; Shenzhen Mindray Bio-Medical Electronics Co., Ltd, Shenzen, China). The 4 remaining blood samples from each participant were used to prepare 4 different PRPs according to the protocols provided by the manufacturers. Four commercial PRP systems that were available on the local medical market were chosen as they are frequently used for the treatment of orthopedic conditions. The systems used included the Arthrex Autologous Conditioned Plasma (ACP) Double Syringe system (Arthrex Inc., Naples, USA), the Mini GPS III Platelet Concentration system (Biomet Inc., Warsaw, USA), the Xerthra PRP kit (Biovico Sp. z o.o., Gdynia, Poland), and Dr. PRP (Rmedica, Seoul, South Korea).

The Arthrex ACP Double Syringe system required $13.5 \mathrm{~mL}$ of whole blood collected into a specially designed double-syringe system within $1.5 \mathrm{~mL}$ ACD-A added as an anticoagulant. The samples were spun at $1500 \mathrm{rpm}$ for $5 \mathrm{~min}$ in a dedicated centrifuge provided by the local distributor. After centrifugation, conditioned plasma in a volume of $4 \mathrm{~mL}$ was transferred to the inner syringe in the double-syringe system and was ready to use.

The Mini GPS III Platelet Concentration system has a specially designed valve for automatic PRP separation. After mixing $27 \mathrm{~mL}$ of whole blood with $3 \mathrm{~mL}$ of ACD-A anticoagulant, the samples were placed in a separator and spun at $3200 \mathrm{rpm}$ for $15 \mathrm{~min}$ in a dedicated centrifuge provided by the local distributor. The platelet-poor plasma was then removed and about $3 \mathrm{~mL}$ of leukocyte-rich (LR)-PRP was collected into a new sterile syringe.

The Xerthra PRP kit required $13.5 \mathrm{~mL}$ of whole blood that was mixed with $1.5 \mathrm{~mL}$ of $3.13 \%$ sodium citrate as an anticoagulant. The samples were spun at $3500 \mathrm{rpm}$ for $5 \mathrm{~min}$ in a dedicated centrifuge provided by the local distributor, transferring plasma into the neck of the tube and removing platelet poor plasma $1.5 \mathrm{~mL}$ of leukocytepoor (LP)-PRP was then collected into a new sterile syringe.

Whole blood in a volume of $18 \mathrm{~mL}$ mixed with $2 \mathrm{~mL}$ of $3.13 \%$ sodium citrate was transferred into the Dr. PRP tube. The samples were then spun for $4 \mathrm{~min}$ at $3100 \mathrm{rpm}$ in a dedicated centrifuge provided by the local distributor. Following this, the piston in the device was used to separate the plasma from the red blood cells (RBC) by moving it into the neck of the tube. After removing platelet poor plasma, LP-PRP in a volume of $3 \mathrm{~mL}$ was collected into a new sterile syringe.

All 24 samples of prepared plasma were analyzed for the content of morphotic components using an automatic laboratory analyzer (Mindray BC-5150). The count and concentration of white blood cells (WBC), RBC and platelets were evaluated immediately after collection of the samples. Platelet capture efficiency (PCE) was calculated using the following formula:

\section{obtained PRP volume $[\mathrm{mL}] \times$ platelets concentration \\ in PRP $(\mathrm{G} / \mathrm{L}) /$ whole blood collected volume $[\mathrm{mL}]$ $\times$ platelets concentration in whole blood $(\mathrm{G} / \mathrm{L})$.}

Following collection, $1 \mathrm{~mL}$ of each PRP sample was placed into an Eppendorf polypropylene tube and went through the platelet activation process developed by Zimmermann et al. ${ }^{22}$ The activation procedure involved freezing at $-80^{\circ} \mathrm{C}$ for $30 \mathrm{~min}$, followed by thawing to room temperature for another $30 \mathrm{~min}$ and freezing for a second time at $-80^{\circ} \mathrm{C}$. The samples were then stored at $-80^{\circ} \mathrm{C}$ until further analysis.

Before cytokine measurement, the PRP samples were thawed completely at room temperature and spun for 5 min at $2.5 \mathrm{rpm}$ using a Micro Star 17 centrifuge (VWR International Company, ThermoElectron LED, Langenselbold, Germany). A custom-designed bead-based multiplex immunoassay that uses fluorescence-encoded beads and flow cytometry (LEGENDplexTM; BioLegend, San Diego, USA) was used to quantify the following platelet growth factors: transforming growth factor- $\beta 1$ (TGF- $\beta 1$, free active), epidermal growth factor (EGF), vascular endothelial growth factor (VEGF), and platelet-derived growth factor-AA (PDGF-AA). The concentration of a particular cytokine was determined by means of a standard curve generated during the performance of the test. The analyses were done according to the manufacturer's instructions. The samples were acquired on CyFlow SPACE and a CyFlow CUBE flow cytometer (Sysmex-Partec, Görlitz, Germany) by applying a $488 \mathrm{~nm}$ laser with a 536/40 (BP) filter for the PE fluorochrome, and a $638 \mathrm{~nm}$ laser with a 675/20 (BP) filter for the APC fluorochrome. The results were analyzed with LEGENDplexTM Data Analysis Software v. 8.0 (Vigene Tech Inc., Carlisle, USA).

All data obtained and analyzed in this study are quantitative. To avoid bias in the obtained results, all PRP samples were made with the same great care.

\section{Statistical methods}

All data were analyzed using STATISTICA v. 13.3 software (StatSoft Inc., Tulsa, USA). Due to the lack of confirmation of to a normal distribution, as assessed with 
the Shapiro-Wilk test, nonparametric methods were used (Kruskal-Wallis one-way analysis of variance (ANOVA) with Dunn's post hoc test and Spearman's correlation). Statistical significance was established at the level of $\mathrm{p}<0.05$.

\section{Results}

\section{Study participants}

Six volunteers, aged $27.8 \pm 0.4$ years, met the criteria for inclusion and were enrolled in the study. The average body mass index (BMI) of the volunteers was $25.82 \pm 2.12 \mathrm{~kg} / \mathrm{m}^{2}$. Each participant signed an informed consent form.

\section{Main results}

\section{Whole blood count}

The distribution of the cellular components of whole blood samples collected from all participants are included in Table 1. All results were in the range of normal physiological values.

\section{Concentration of platelets}

The platelet concentrations for the PRP samples are illustrated in Fig. 1. Only 1 single sample of PRP delivered by Xerthra PRP kit had a lower concentration of platelets than baseline. The highest platelet concentration was produced with the Mini GPS III System $\left(1266.33 \pm 347.96 \times 10^{9} / \mathrm{L}\right)$, and the lowest with Arthrex ACP $\left(395 \pm 110.15 \times 10^{9} / \mathrm{L}\right)$. Statistical analysis showed a significant difference between the 2 systems mentioned above ( $\mathrm{p}=0.003$ ), and between the Mini GPS III and Xerthra PRP kits $\left(513.67 \pm 255.99 \times 10^{9} / \mathrm{L} ; \mathrm{p}=0.04\right)$. There was no significant difference when compared to Dr. PRP $\left(504.83 \pm 106.29 \times 10^{9} / \mathrm{L} ; \mathrm{p}>0.05\right)$.

\section{PCE}

The PCE results are illustrated in Fig. 2. The highest PCE score was obtained from the Mini GPS III system (53.76 $\pm 6.66 \%)$, and the lowest from the Xerthra PRP kit

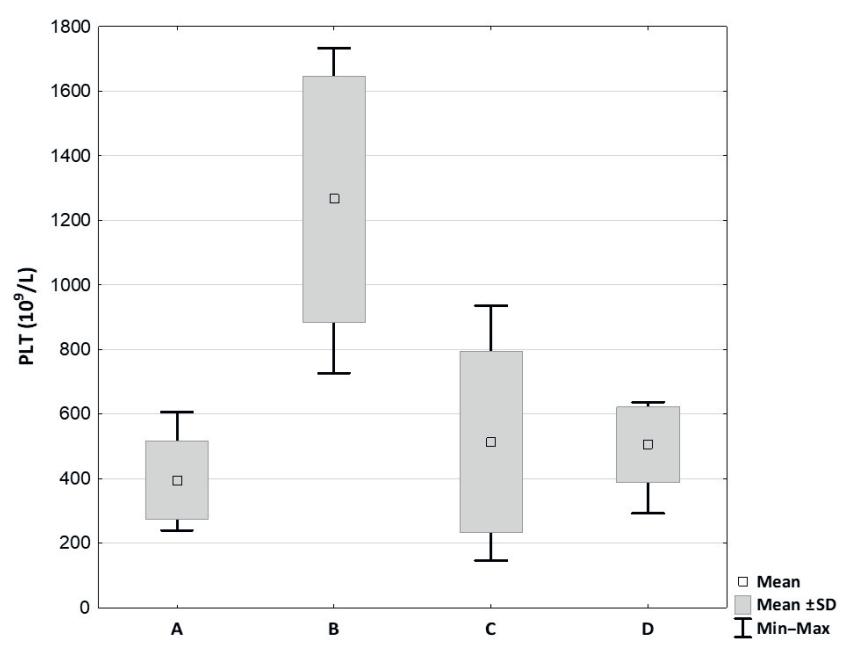

Fig. 1. Platelet concentration in PRP obtained using different systems A - Arthrex ACP; B - Mini GPS III System; C - Xerthra PRP kit; D - Dr. PRP.

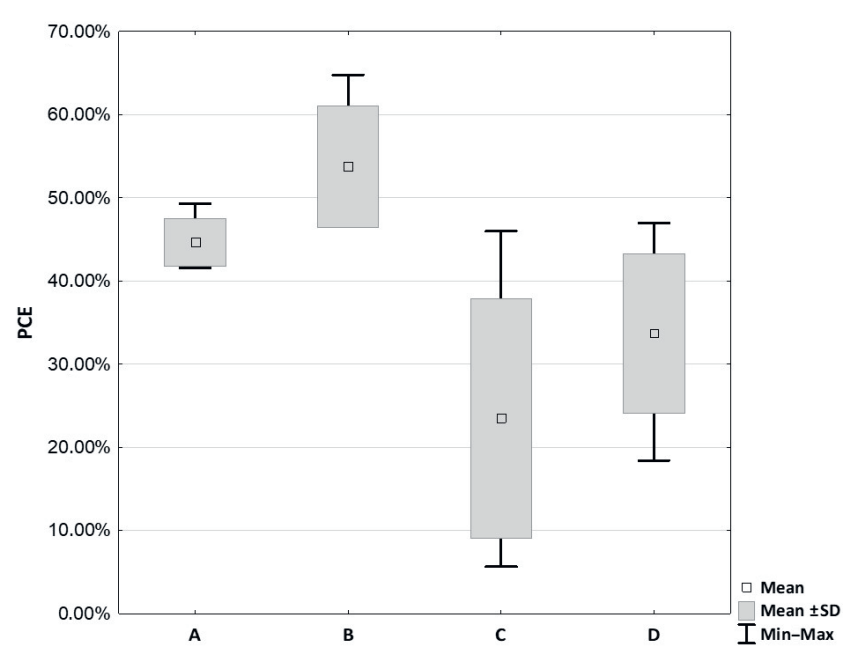

Fig. 2. Platelet capture efficiency in PRP obtained using different systems A - Arthrex ACP; B - Mini GPS III System; C - Xerthra PRP kit; D - Dr. PRP.

(23.50 $\pm 13.13 \%)$. The Mini GPS III System provided a significantly higher PCE then the Xerthra PRP kit ( $\mathrm{p}=0.001)$ and Dr. PRP (33.68 $\pm 8.78 \%$; $=0.02)$. Although the PCE

Table 1. Whole blood characteristics from all participants (NR 1-6)

\begin{tabular}{|c|c|c|c|c|c|c|c|}
\hline \multirow{2}{*}{ Blood parameter } & \multicolumn{6}{|c|}{ Participant } & \multirow{2}{*}{ Mean } \\
\hline & NR1 & NR2 & NR3 & NR4 & NR5 & NR6 & \\
\hline $\operatorname{RBC}\left[10^{12} / L\right]$ & 4.69 & 4.47 & 4.97 & 5.82 & 5.5 & 5.04 & $5.08 \pm 0.46$ \\
\hline Platelets $\left[10^{9} / \mathrm{L}\right]$ & 171 & 288 & 244 & 226 & 364 & 264 & $259.5 \pm 59.09$ \\
\hline WBC $\left[10^{9} / L\right]$ & 4.79 & 5.62 & 7.22 & 6.5 & 7.64 & 6.19 & $6.33 \pm 0.95$ \\
\hline Neutrophils $\left[10^{9} / \mathrm{L}\right]$ & 2.52 & 3.01 & 4.13 & 3.34 & 4.37 & 2.97 & $3.39 \pm 0.66$ \\
\hline Lymphocytes $\left[10^{9} / L\right]$ & 1.79 & 1.83 & 2.41 & 2.44 & 2.53 & 2.53 & $2.26 \pm 0.32$ \\
\hline Monocytes $\left[10^{9} / \mathrm{L}\right]$ & 0.34 & 0.4 & 0.53 & 0.49 & 0.54 & 0.45 & $0.46 \pm 0.07$ \\
\hline Eosinophils $\left[10^{9} / \mathrm{L}\right]$ & 0.11 & 0.34 & 0.11 & 0.18 & 0.17 & 0.21 & $0.19 \pm 0.08$ \\
\hline Basophils $\left[10^{9} / \mathrm{L}\right]$ & 0.03 & 0.04 & 0.04 & 0.05 & 0.03 & 0.03 & $0.04 \pm 0.01$ \\
\hline
\end{tabular}

RBC - red blood cells; WBC - white blood cells. 
obtained from the Arthrex ACP kit (44.66 $\pm 2.65 \%)$ was lower than that for the Mini GPS III and higher than that for the Xerthra PRP and Dr. PRP kits, the differences were not statistically significant $(\mathrm{p}=0.061, \mathrm{p}=0.22, \mathrm{p}=1$, respectively).

\section{Concentration of WBC}

The WBC concentrations for the PRP samples are illustrated in Fig. 3. The highest concentrations of WBC were obtained with the Mini GPS III system $\left(34.81 \pm 9.59 \times 10^{9} / \mathrm{L}\right)$, and it was the only system that produced a WBC concentration above the whole blood baseline level. Statistical analysis showed significant differences when comparing Mini GPS III to Arthrex ACP $\left(0.78 \pm 0.73 \times 10^{9} / \mathrm{L}\right.$; $\mathrm{p}=0.02)$ and Dr. PRP $\left(0.50 \pm 0.59 \times 10^{9} / \mathrm{L} ; \mathrm{p}=0.001\right)$, but not to the Xerthra PRP kit $\left(1.91 \pm 1.87 \times 10^{9} / \mathrm{L} ; \mathrm{p}=0.16\right)$.

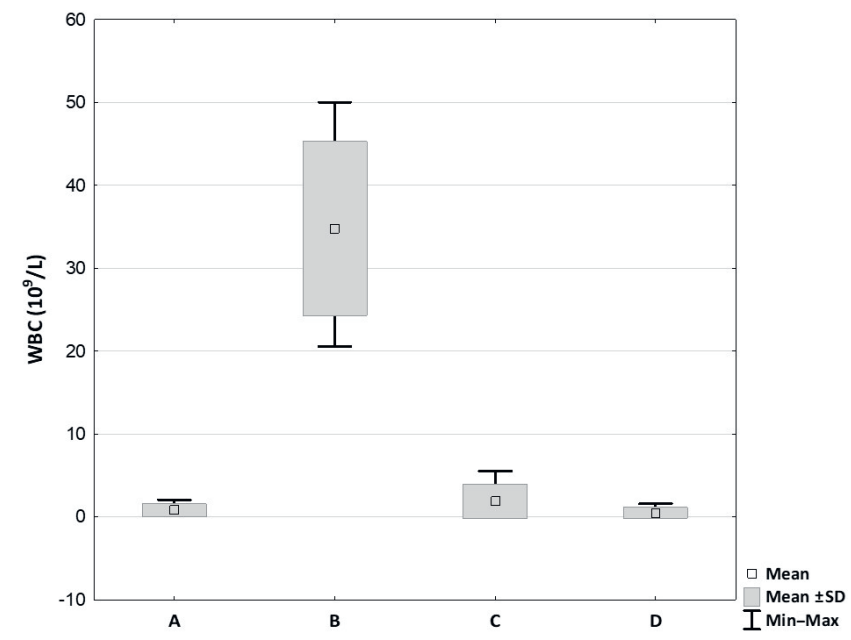

Fig. 3. White blood cells concentration in PRP obtained using different systems

A - Arthrex ACP; B - Mini GPS III System; C - Xerthra PRP kit; D - Dr. PRP.

\section{Concentration of RBC}

The RBC concentrations for the PRP samples are illustrated in Fig. 4. One of the goals of producing PRP is to separate it from plasma containing $\mathrm{RBC}$. Hence, the RBC concentration in PRP can be treated as a measure of purification. The Mini GPS III System delivered PRP with the highest concentration of RBC $\left(1.48 \pm 0.88 \times 10^{12} / \mathrm{L}\right)$, which was significantly higher than

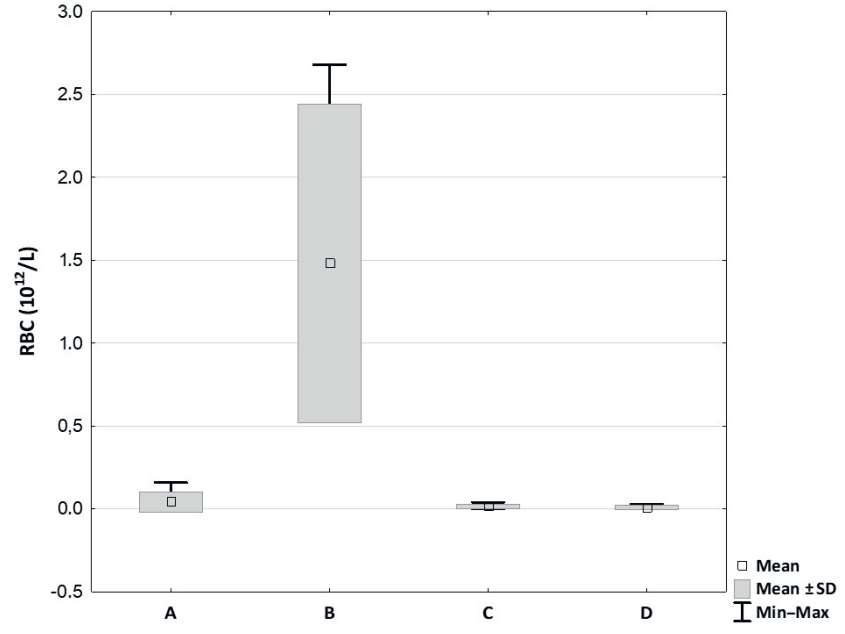

Fig. 4. Red blood cells concentration in PRP obtained using different systems

A - Arthrex ACP; B - Mini GPS III System; C - Xerthra PRP kit; D - Dr. PRP.

Arthrex ACP $\left(0.04 \pm 0.06 \times 10^{12} / \mathrm{L} ; \mathrm{p}=0.045\right)$, the Xerthra PRP kit $\left(0.02 \pm 0.01 \times 10^{12} / \mathrm{L} ; \mathrm{p}=0.04\right)$ and Dr. PRP $\left(0.01 \pm 0.01 \times 10^{12} / \mathrm{L} ; \mathrm{p}=0.004\right)$. No significant differences were found between the other systems.

\section{Concentration of growth factors}

All obtained growth factor concentrations are included in Table 2. Statistical analysis showed that the Mini GPS III System compared to Arthrex ACP delivered PRP with significantly higher levels of EGF (364.1 $\pm 180.16 \mathrm{pg} / \mathrm{mL}$ compared to $107.37 \pm 95.12 \mathrm{pg} / \mathrm{mL}$; $\mathrm{p}=0.04)$ and PDGF-AA $(98,698 \pm 23,843.58 \mathrm{pg} / \mathrm{mL}$ compared to $33,172.5 \pm 13,266.38 \mathrm{pg} / \mathrm{mL} ; \mathrm{p}=0.02)$. There were no significant differences among the other systems and growth factors.

\section{Correlation between growth factors and morphotic components}

All Spearman's correlations are presented in Fig. 5. There was a significant positive correlation between platelet concentration and both EGF and PDFG-AA (Spearman's $R$ values 0.46 and 0.58 , respectively). A significant positive correlation was also observed between the WBC concentration and PDFG-AA (Spearman's R value 0.51). The RBC concentration was also significantly positively correlated

Table 2. Concentration of growth factors in PRP obtained using different systems

\begin{tabular}{|c|c|c|c|c|}
\hline \multirow{2}{*}{ System } & \multicolumn{4}{|c|}{ Growth factor } \\
\hline & TGF- $\beta 1[\mathrm{pg} / \mathrm{mL}]$ & $\mathrm{EGF}[\mathrm{pg} / \mathrm{mL}]$ & VEGF [pg/mL] & PDGF-AA [pg/mL] \\
\hline Arthrex ACP & $58.12 \pm 76.92$ & $107.37 \pm 95.12$ & $138.88 \pm 189.52$ & $33172.5 \pm 13266.38$ \\
\hline Mini GPS III System & $31.72 \pm 17.26$ & $364.1 \pm 180.16$ & $456.06 \pm 301.51$ & $98698 \pm 23843.58$ \\
\hline Xerthra PRP kit & $45.97 \pm 59.60$ & $161.20 \pm 125.34$ & $288.61 \pm 364.54$ & $54565.43 \pm 43241.12$ \\
\hline Dr. PRP & $30.18 \pm 25$ & $223.48 \pm 173.63$ & $187.58 \pm 134.90$ & $41400.6 \pm 18537.8$ \\
\hline
\end{tabular}

TGF- $\beta 1$ - transforming growth factor- $\beta 1$; EGF - epidermal growth factor; VEGF - vascular endothelial growth factor; PDGF-AA - platelet-derived growth factor-AA. 

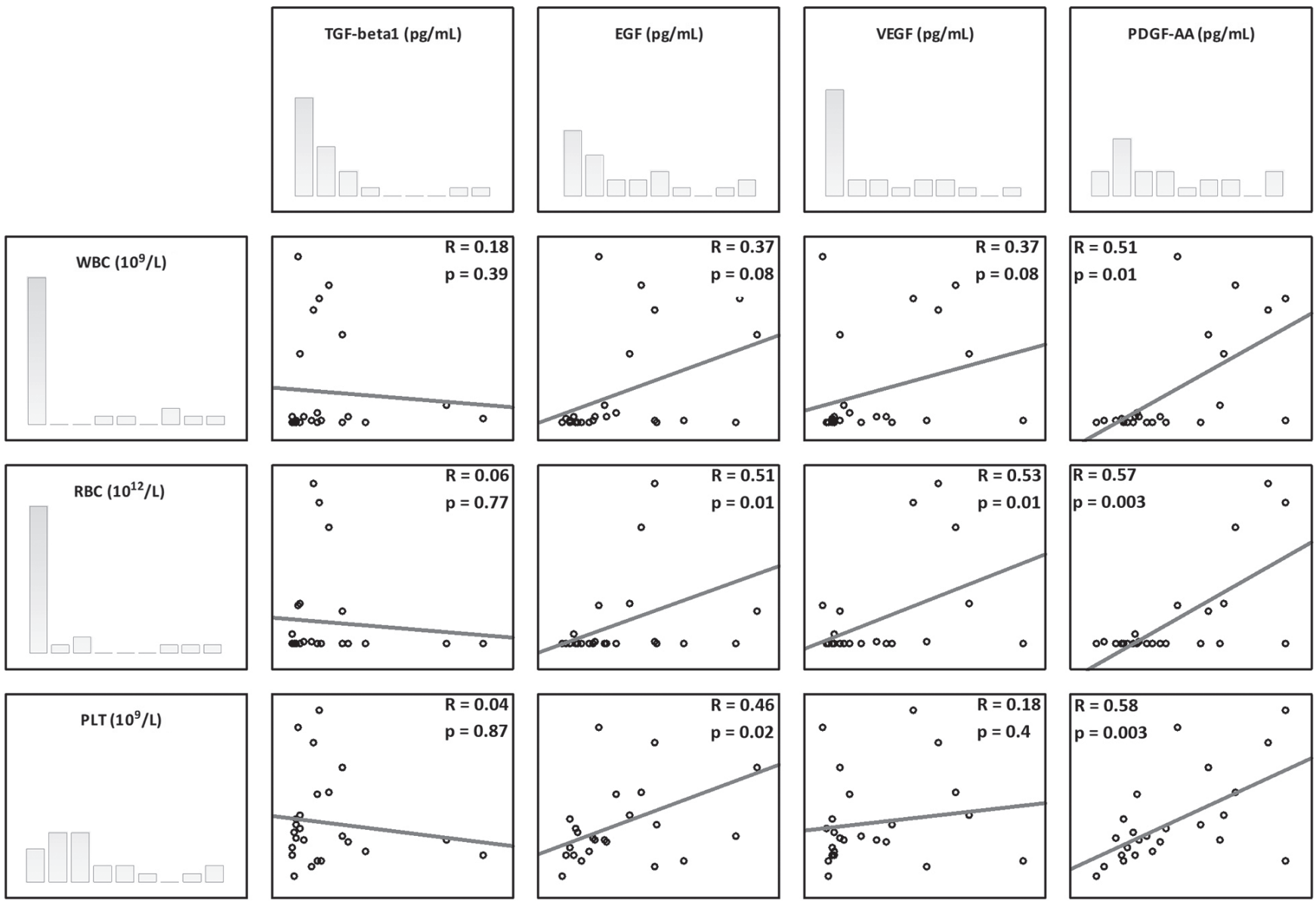

Fig. 5. Spearman correlations between morphotic components and growth factors in PRP samples

with the levels of EGF, VEGF and PDGF-AA (Spearman's $R$ values $0.51,0.53$ and 0.57 , respectively). No significant correlation was found between TGF- $\beta 1$ and any PRP morphotic component.

\section{Discussion}

As expected, the current study showed differences between PRP produced with the use of various commercial kits. These findings confirm the results of other studies that have been summarized in a recent systemic review. ${ }^{19}$ However, it is important to point out that these differences do not determine the possible clinical superiority of any of the described systems.

All kits used in the current study produced PRP with an average platelet concentration above the whole blood baseline level. For unexplained reasons, 1 PRP sample obtained using the Xerthra PRP system had a platelet concentration below the baseline level. The Mini GPS III System produced the highest platelet concentration in relation to the whole blood baseline level $(\times 4.84 \pm 0.6)$, while the lowest concentration was generated with the Arthrex ACP system $(\times 1.51 \pm 0.09)$. These 2 systems are among the most studied and others have reported similar results for platelet concentrations. ${ }^{17,19,23,24}$ The Xerthra PRP kit and Dr. PRP were able to concentrate platelets in similar manner $(\times 2.11 \pm 1.18$ and $\times 2.02 \pm 0.53$, respectively).

Both too low and too high a platelet concentration can reduce the chances of a good therapeutic response. Platelet levels below the baseline are not sufficient to induce a significant response and a concentration above $\times 6$ may slow down the repair processes. ${ }^{21}$ Graziani et al. concluded that platelet concentrations of approx. 2.5 times greater than native blood achieved a maximum effect on osteoblast and fibroblast proliferation in vitro. In addition, higher dosages 3.5 times above baseline could lead to some adverse events. ${ }^{25}$ Various studies have also reported different ideal therapeutic platelet concentrations. While some authors recommend a platelet concentration of about $1000 \times 10^{3} / \mathrm{mL}$, others consider a number $>200 \times 10^{3} / \mathrm{mL}$ as sufficient. ${ }^{26,27}$ According to this latter definition of PRP, all samples included in this study met the criteria. ${ }^{28}$

In the current study, there were significant correlations observed between platelet concentration and both EGF and PDGF-AA, but no correlations with VEGF and TGF- $\beta 1$. These results are somewhat different from what has been reported previously. For example, Magalon observed significant correlations between platelet dose and all growth factors examined (VEGF, EGF, PDGF-AB, and 
TGF- $\beta 1) .{ }^{17}$ Similarly, Sundman also reported positive significant correlations between platelets and both TGF- $\beta 1$ and PDGF-AB. ${ }^{29}$

Significantly higher concentrations of WBC were delivered by the Mini GPS III system as it is designed for LR-PRP production. The mean concentration of WBC produced by this system was $5.55 \pm 1.65$ times above the baseline in whole blood. The mean content of neutrophils and leukocytes were distributed almost equally (15.72 \pm 8.11 $\times 10^{9} / \mathrm{L}$ and $15.99 \pm 2.96 \times 10^{9} / \mathrm{L}$, respectively). Similar results have been observed in previous studies. ${ }^{17-19,23,24,29} \mathrm{Ar}-$ threx ACP, Xerthra PRP kit and Dr. PRP delivered LP-PRP with a WBC concentration much lower than the baseline level. According to the literature, the presence of leukocytes in PRP could affect the levels of important growth factors such as VEGF and EGF, and may also have antibacterial or immune-regulating effects. ${ }^{17,18,30}$ While we did not observe a correlation between WBC and both VEGF and EGF, a positive correlation with PDGF-AA was found. This finding has not been reported in previous research, likely because PDGF-AA is not a frequently analyzed cytokine. In vitro studies have shown a potential negative effect on the healing of tendon structures due to the high content of proteinases and hydrolases in WBC, especially in neutrophils. ${ }^{29,31-33}$ In vivo studies do not confirm this effect; however, when planning the therapy for enthesopathies, one should take into consideration the possible stimulation of catabolic processes by a high content of leukocytes. ${ }^{15,21}$

An efficient PRP preparation procedure should remove $\mathrm{RBC}$ as much as possible, as their presence is considered as the sign of impurity. This is likely the reason why the most studies do not analyze the correlation between RBC and growth factors. Our results showed a significant correlation between RBC concentration and levels of growth factors such as EGF, VEGF and PDGF-AA. The strength of correlation was moderate (Spearman's R value between 0.51 and 0.57 ) but still worthy of further analysis in a larger population. Among the tested PRP preparation kits, the Mini GPS III System produced a significantly higher RBC contamination compared to other systems. This may be one of the factors behind the higher growth factor content in PRP obtained by the Mini GPS III System in other studies. ${ }^{17,19}$ Arthrex ACP, Xerthra PRP kit and Dr. PRP delivered PRP with an almost undetectable RBC concentration.

\section{Limitations}

To date, numerous studies have already shown the diversity in the morphotic components and growth factor content in PRP obtained various using methods. However, systems such as the Xerthra PRP kit and Dr. PRP were tested here for the first time. There are many more commercially available systems for PRP preparation than those included in the study. Even if we chose the most popular systems, it does not allow the results to be transferred to other systems, and they have to be evaluated separately.
As this is a preliminary study, we decided to examine only a few selected growth factors. Many other cytokines, chemokines and growth factors released by platelets are also involved in tissue healing. The task for future research is to study all of the cytokines that play a key role in regenerative processes. On the other hand, even a precise determination of the cytokine content does not allow one to predict clinical effects in vivo. Therefore, it will be necessary to evaluate how differences in PRP characteristics affect living tissues.

Due to the lack of confirmation to a normal distribution for most of the data, nonparametric methods were used for statistical analyses. This lack of confirmation was likely due to the small sample size used, and extension to a larger group of participants may provide more reliable results.

\section{Conclusions}

The current study showed a wide heterogeneity in the characteristics of autologous PRPs produced by various commercial kits. Based on the obtained results and previous studies, the correlation between the concentration of desired growth factors and morphotic components remains unclear. Due to insufficient data, we cannot argue for or against use of any of the mentioned protocols for the treatment of enthesopathy. Further studies on a larger population that examine a wider variety of cytokines are required to validate our results. Future research should also focus on both the in vitro and in vivo biological effects of PRP produced by different preparation protocols to establish the effects of different concentrations of the various growth factors on tissue healing.

\section{ORCID iDs}

Maciej Dejnek (D) https://orcid.org/0000-0002-6675-0256 Helena Moreira (iD https://orcid.org/0000-0002-8084-3686 Sylwia Płaczkowska (1) https://orcid.org/0000-0002-1466-3820 Piotr Morasiewicz (D) https://orcid.org/0000-0002-7587-666X Ewa Barg (D) https://orcid.org/0000-0002-7069-5026 Jarosław Witkowski (i) https://orcid.org/0000-0002-2754-1339 Paweł Reichert (D) https://orcid.org/0000-0002-0271-4950

\section{References}

1. Fitzpatrick J, Bulsara M, Zheng MH. The effectiveness of platelet-rich plasma in the treatment of tendinopathy. Am J Sports Med. 2017;45(1): 226-233. doi:10.1177/0363546516643716

2. Mi B, Liu G, Zhou W, et al. Platelet rich plasma versus steroid on lateral epicondylitis: Meta-analysis of randomized clinical trials. Phys Sportsmed. 2017;45(2):97-104. doi:10.1080/00913847.2017.1297670

3. Królikowska A, Sikorski Ł, Czamara A, Reichert P. Are the knee extensor and flexor muscles isokinetic parameters affected by the duration of postoperative physiotherapy supervision in patients eight months after $A C L$ reconstruction with the use of semitendinosus and gracilis tendons autograft? Acta Bioeng Biomech. 2018;20(4):89-100. doi:10.5277/ABB-01149-2018-02

4. Sánchez-González DJ, Méndez-Bolaina E, Trejo-Bahena NI. Plateletrich plasma peptides: Key for regeneration. Int J Pept. 2012;2012:532519. doi:10.1155/2012/532519.

5. Creaney L, Hamilton B. Growth factor delivery methods in the management of sports injuries: The state of play. Br J Sports Med. 2008; 42(5):314-320. doi:10.1136/bjsm.2007.040071 
6. Werner S, Grose R. Regulation of wound healing by growth factors and cytokines. Physiol Rev. 2003;83(3):835-870. doi:10.1152/physrev. 2003.83.3.835

7. Molloy T, Wang Y, Murrell GAC. The roles of growth factors in tendon and ligament healing. Sport Med. 2003;33(5):381-394. doi:10.2165/ 00007256-200333050-00004

8. Alsousou J, Thompson M, Hulley P, Noble A, Willett K. The biology of platelet-rich plasma and its application in trauma and orthopaedic surgery: A review of the literature. J Bone Joint Surg Br. 2009;91(8): 987-996. doi:10.1302/0301-620X.91B8.22546

9. Tenore G, Zimbalatti A, Rocchetti F, et al. Management of medication-related osteonecrosis of the kaw (MRONJ) using leukocyte- and platelet-rich fibrin (L-PRF) and photobiomodulation: A retrospective study. J Clin Med. 2020;9(11):3505. doi:10.3390/jcm9113505

10. Suthar M, Gupta S, Bukhari S, Ponemone V. Treatment of chronic nonhealing ulcers using autologous platelet rich plasma: A case series. J Biomed Sci. 2017;24(1):16. doi:10.1186/s12929-017-0324-1

11. Tietze DC, Geissler K, Borchers J. The effects of platelet-rich plasma in the treatment of large-joint osteoarthritis: A systematic review. Phys Sportsmed. 2014;42(2):27-37. doi:10.3810/psm.2014.05.2055

12. Moraes VY, Lenza M, Tamaoki MJ, Faloppa F, Belloti JC. Platelet-rich therapies for musculoskeletal soft tissue injuries. Cochrane Database Syst Rev. 2013;12:CD010071. doi:10.1002/14651858.CD010071.pub2

13. Scott A, LaPrade RF, Harmon KG, et al. Platelet-rich plasma for patellar tendinopathy: A randomized controlled trial of leukocyte-rich PRP or leukocyte-poor PRP versus saline. Am J Sports Med. 2019;47(7): 1654-1661. doi:10.1177/0363546519837954

14. Navani A, Li G, Chrystal J. Platelet rich plasma in musculoskeletal pathology: A necessary rescue or a lost cause? Pain Physician. 2017; 20(3):E345-E356. doi:10.36076/ppj.2017.e356

15. Wasterlain AS, Braun HJ, Dragoo JL. Contents and formulations of platelet-rich plasma. Oper Tech Orthop. 2012;22(1):33-42. doi:10.1053/ j.oto.2011.11.001

16. Kushida S, Kakudo N, Morimoto N, et al. Platelet and growth factor concentrations in activated platelet-rich plasma: A comparison of seven commercial separation systems. J Artif Organs. 2014;17(2): 186-192. doi:10.1007/s10047-014-0761-5

17. Magalon J, Bausset O, Serratrice N, et al. Characterization and comparison of 5 platelet-rich plasma preparations in a single-donor model. Arthroscopy. 2014;30(5):629-638. doi:10.1016/j.arthro.2014.02.020

18. Castillo TN, Pouliot MA, Kim HJ, Dragoo JL. Comparison of growth factor and platelet concentration from commercial platelet-rich plasma separation systems. Am J Sports Med. 2011;39(2):266-271. doi:10.1177/0363546510387517

19. Oudelaar BW, Peerbooms JC, Huis In 't Veld R, Vochteloo AJH. Concentrations of blood components in commercial platelet-rich plasma separation systems: A review of the literature. Am J Sports Med. 2019;47(2):479-487. doi:10.1177/0363546517746112

20. Dohan Ehrenfest DM, Rasmusson L, Albrektsson T. Classification of platelet concentrates: From pure platelet-rich plasma (P-PRP) to leucocyte- and platelet-rich fibrin (L-PRF). Trends Biotechnol. 2009;27(3): 158-167. doi:10.1016/j.tibtech.2008.11.009
21. Delong JM, Russell RP, Mazzocca AD. Platelet-rich plasma: The PAW classification system. Arthroscopy. 2012;28(7):998-1009. doi:10.1016/j. arthro.2012.04.148

22. Zimmermann R, Arnold D, Strasser E, et al. Sample preparation technique and white cell content influence the detectable levels of growth factors in platelet concentrates. Vox Sang. 2003;85(4):283-289. doi:10.1111/j.0042-9007.2003.00361.x

23. Oh JH, Kim WOO, Park KU, Roh YH. Comparison of the cellular composition and cytokine-release kinetics of various platelet-rich plasma preparations. Am J Sports Med. 2015;43(12):3062-3070. doi:10. $1177 / 0363546515608481$

24. Mazzocca AD, McCarthy MB, Chowaniec DM, et al. Platelet-rich plasma differs according to preparation method and human variability. J Bone Joint Surg Am. 2012;94(4):308-316. doi:10.2106/JBJS.K.00430

25. Graziani F, Ivanovski S, Cei S, Ducci F, Tonetti M, Gabriele M. The in vitro effect of different PRP concentrations on osteoblasts and fibroblasts. Clin Oral Implants Res. 2006;17(2):212-219. doi:10.1111/j.1600-0501. 2005.01203.x

26. Marx RE. Platelet-rich plasma (PRP): What is PRP and what is not PRP? Implant Dent. 2001;10(4):225-228. doi:10.1097/00008505-20011000000002

27. Mazzucco L, Balbo V, Cattana E, Guaschino R, Borzini P. Not every PRP-gel is born equal. Evaluation of growth factor availability for tissues through four PRP-gel preparations: Fibrinet ${ }^{\circledR}$, RegenPRP-Kit $^{\circledR}$, Plateltex ${ }^{\circledR}$ and one manual procedure. Vox Sang. 2009;97(2):110-118. doi:10.1111/j.1423-0410.2009.01188.x

28. Królikowska A, Reichert P, Czamara A, Krzemińska K. Peak torque angle of anterior cruciate ligament-reconstructed knee flexor muscles in patients with semitendinosus and gracilis autograft is shifted towards extension regardless of the postoperative duration of supervised physiotherapy. PLoS One. 2019;14(2):e0211825. doi: 10.1371/journal. pone.0211825

29. Sundman EA, Cole BJ, Fortier LA. Growth factor and catabolic cytokine concentrations are influenced by the cellular composition of platelet-rich plasma. Am J Sports Med. 2011;39(10):2135-2140. doi:10. $1177 / 0363546511417792$

30. Cieślik-Bielecka A, Reichert P, Skowroński R, Królikowska A, Bielecki T. A new aspect of in vitro antimicrobial leukocyte- and platelet-rich plasma activity based on flow cytometry assessment. Platelets. 2019; 30(6):728-736. doi:10.1080/09537104.2018.1513472

31. Kobayashi $Y$, Saita $Y$, Nishio H, et al. Leukocyte concentration and composition in platelet-rich plasma (PRP) influences the growth factor and protease concentrations. J Orthop Sci. 2016;21(5):683-689. doi:10.1016/j.jos.2016.07.009

32. McCarrel TM, Minas T, Fortier LA. Optimization of leukocyte concentration in platelet-rich plasma for the treatment of tendinopathy. J Bone Joint Surg Am. 2012;94(19):e143(1). doi:10.2106/JBJS.L.00019

33. Zhang $L$, Chen $S$, Chang P, et al. Harmful effects of leukocyte-rich platelet-rich plasma on rabbit tendon stem cells in vitro. Am J Sports Med. 2016;44(8):1941-1951. doi:10.1177/0363546516644718 